\title{
Hydrogen infrared recombination lines as a diagnostic tool for the geometry of the circumstellar material of hot stars ${ }^{\star}$
}

\author{
A. Lenorzer ${ }^{1}$, A. de Koter ${ }^{1}$, and L. B. F. M. Waters ${ }^{1,2}$ \\ 1 Astronomical Institute "Anton Pannekoek", University of Amsterdam, Kruislaan 403, 1098 SJ Amsterdam, \\ The Netherlands \\ 2 Instituut voor Sterrenkunde, K.U. Leuven, Celestijnenlaan 200B, 3001 Heverlee, Belgium
}

Received 24 January 2002 / Accepted 1 March 2002

\begin{abstract}
We have analysed the infrared hydrogen recombination lines of a sample of well studied hot massive stars observed with the Infrared Space Observatory. Our sample contains stars from several classes of objects, whose circumstellar environment is believed to be dominated by an ionized stellar wind (the Luminous Blue Variables) or by a dense disk-like geometry (Be stars and B[e] stars). We show that hydrogen infrared recombination lines can be used as a diagnostic tool to constrain the geometry of the ionized circumstellar material. The line strengths are sensitive to the density of the emitting gas. High densities result in optically thick lines for which line strengths are only dependent on the emitting surface. Low density gas produces optically thin lines which may be characterized by Menzel case B recombination. The ISO observations show that stellar winds are dominated by optically thin H I recombination lines, while disks are dominated by optically thick lines. Disks and winds are well separated in a diagnostic diagram using the $\mathrm{Hu}(14-6) / \mathrm{Br} \alpha$ and the $\mathrm{Hu}(14-6) / \mathrm{Pf} \gamma$ line flux ratios. This diagnostic tool is useful to constrain the nature of hot star environments in case they are highly obscured, for instance while they are still embedded in their natal molecular cloud.
\end{abstract}

Key words. stars: circumstellar matter - early-type - emission-line - mass loss - winds

\section{Introduction}

Hot massive stars are characterized by the presence of ionized circumstellar matter, which is in the majority of cases due to a stellar wind. The presence of this ionized gas is easily detected at ultraviolet wavelengths through $\mathrm{P}$ Cygni profiles of resonance lines of e.g. C IV, Si IV and $\mathrm{N} v$, while at optical and infrared wavelengths free-bound and free-free continuum emission as well as $\mathrm{H}$ I recombination line radiation can be observed. Both the strength and the shape of the lines formed in the circumstellar gas are sensitive to the densities, velocities and the geometry of the gas. For instance, the $\mathrm{P}$ Cygni profiles in $\mathrm{H} \alpha$ seen in hot stars with dense winds are indicative of a roughly spherical outflow whose terminal velocity can be derived from the blue edge of the absorption part of the profile (e.g. Castor \& Lamers 1979). The ionized disks surrounding Be stars show a characteristic double-peaked emission line structure, and can be explained by almost Keplerian

Send offprint requests to: A. Lenorzer,

e-mail: lenorzer@astro.uva.nl

* Based on observations with ISO, an ESA project with instruments funded by ESA Member States (especially the PI countries: France, Germany, The Netherlands and the UK) and with the participation of ISAS and NASA. rotation with hardly any radial expansion (Struve 1933; Limber \& Marlborough 1968). The situation is less clear in the case of the so-called B[e] stars, partly due to the mixed nature of the stars in this group (see Lamers et al. 1998). However, most of these stars are believed to be surrounded by a flattened envelope.

While the wind diagnostics at UV and optical wavelengths are well studied and an extensive literature exists, the situation is less well documented at infrared wavelengths. This is mostly due to the unavailability, until recently, of high quality infrared spectra. As infrared instrumentation becomes more sensitive, the stellar population of highly obscured regions becomes accessible, such as the galactic centre or star forming regions. Since traditional wind diagnostics are no longer usable in these regions, infrared observations must be used to characterize the stellar populations and their circumstellar matter.

In this Letter, we present a simple diagnostic diagram which allows one to constrain the geometry of the ionized part of the circumstellar envelope of hot stars by means of measuring line flux ratios in a few well-chosen H I recombination lines. We calibrate this new diagnostic tool using well-studied, and optically bright hot stars observed with the Short Wavelength Spectrometer (SWS, 
de Graauw et al. 1996) on board of the Infrared Space Observatory (ISO, Kessler et al. 1996). The ISO spectra we use were presented by Lenorzer et al. (2002).

This paper is organized as follows: in Sect. 2 we introduce the sample of stars and the observations; Sect. 3 discusses the line fluxes and the $\mathrm{Hu}(14-6) / \mathrm{Br} \alpha$ versus $\mathrm{Hu}(14-6) / \mathrm{Pf} \gamma$ diagram. Section 4 summarizes the results of this paper.

\section{The sample of stars and the ISO spectra}

The ISO database contains infrared spectra of a wide range of hot stars, observed in different programmes. In Lenorzer et al. (2002) a homogeneous sample of ISO-SWS hot star spectra is presented, and line fluxes are derived. We use the results of that study in the present analysis. All stars discussed here were observed in at least the 2.4-4.1 $\mu \mathrm{m}$ spectral region (band 1 of SWS; de Graauw et al. 1996). Figure 1 displays representative spectra of the three classes of stars to which our diagnostic can be applied (i.e. $\mathrm{Hu}(14-6)$ can be measured): the Luminous Blue Variables (LBVs), the B[e] stars and the Be stars. Before discussing the ISO spectra, we briefly summarize the main properties of these three classes of objects.

$L B V s$ are a rare class of hot massive post-mainsequence stars characterized by a dense ionized stellar wind $\left(\dot{M} \approx 10^{-5} M_{\odot} /\right.$ yr $)$ expanding at modest speed $\left(v_{\exp }=100-300 \mathrm{~km} \mathrm{~s}^{-1}\right)$ (Humphreys \& Davidson 1994). The LBVs show variability on a range of timescales and amplitudes, whose nature is not understood (e.g. van Genderen 2001). Recent high quality observations in the radio (Skinner et al. 1997) and in $\mathrm{H} \alpha$ (Vakili et al. 1997; Chesneau et al. 2000) show that the wind of P Cygni is variable and clumpy, however its overall geometry does not strongly differ from spherical. The near-IR spectrum of LBV's is dominated by free-bound and free-free emission from the wind, as well as by strong recombination lines of $\mathrm{H}$ and He. Many LBVs also show forbidden lines indicative of an extended low-density region. LBVs are known for the presence of extended, mostly dusty nebulae that are the result of large mass ejection in the (recent) past.

$B[e]$ stars are a poorly characterized class of hot stars surrounded by large amounts of ionized and neutral/molecular material. The luminosity class of B[e] stars can range from dwarf to supergiant. At infrared wavelengths, a large excess due to the presence of hot circumstellar dust is observed (e.g. Swings 1974); in addition, hot CO gas is detected in some stars (MgGregor et al. 1989). B[e] stars have prominent H I recombination lines and strong forbidden lines. Spectropolarimetric observations suggest that the circumstellar material is not distributed spherically symmetric, but rather disk-like (see e.g. Bjorkman et al. 1998).

$B e$ stars are rapidly rotating $\mathrm{B}$ type dwarfs or giants that show, or have shown, $\mathrm{H} \alpha$ emission. The $\mathrm{H} \alpha$ line is often double-peaked and the line width scales with the projected rotational velocity of the photosphere (see e.g.

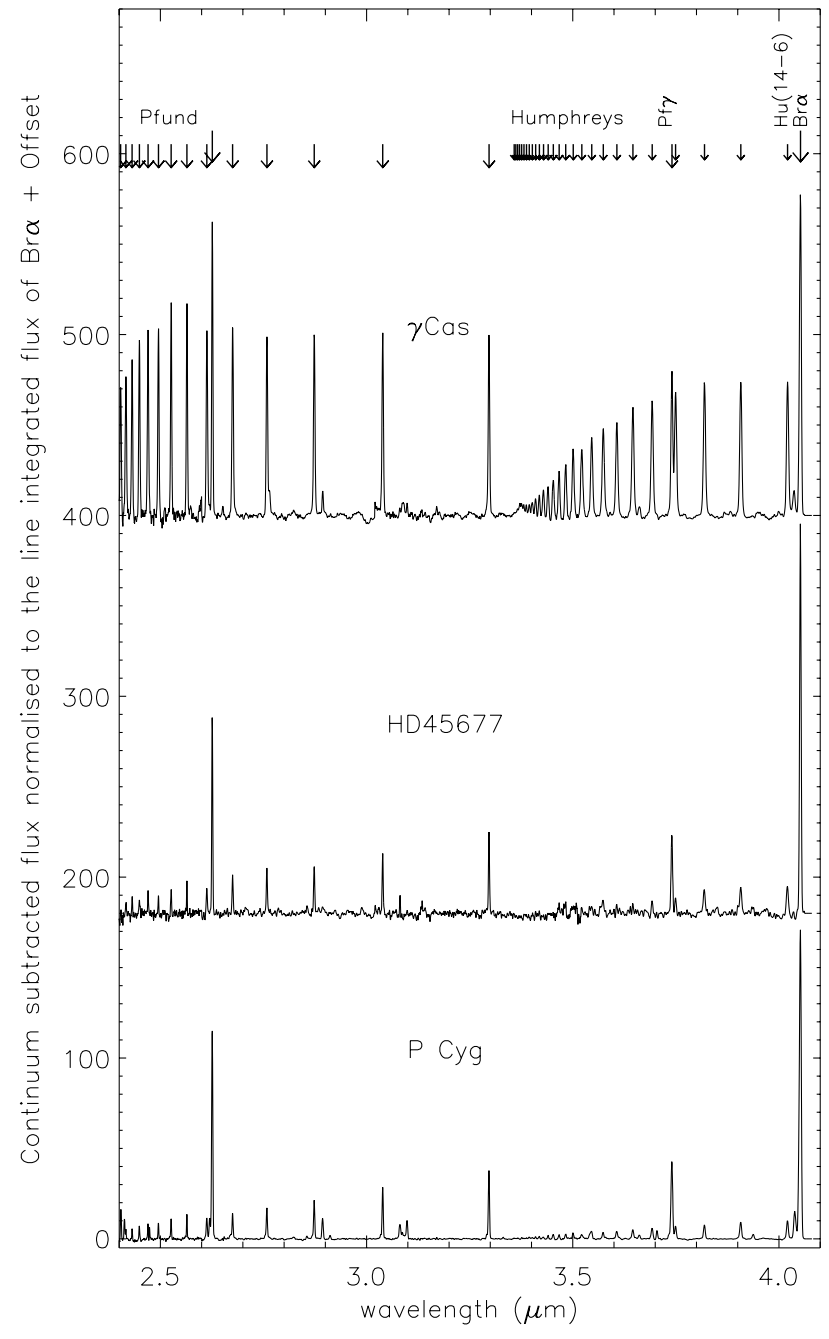

Fig. 1. Typical examples of the three classes of hot star IR spectra considered. Top spectrum: the Be star $\gamma$ Cas; middle spectrum: the $\mathrm{B}[\mathrm{e}]$ star HD 45677; lower spectrum: the LBV P Cygni (see text for discussion).

Dachs et al. 1986). The infrared spectrum is dominated by a large excess due to ionized circumstellar gas of high density, as well as by strong $\mathrm{H}$ I (and, for the hottest Be stars, He I) recombination line emission. Interferometric, direct imaging as well as polarimetric observations show that the gas must be located in a highly flattened circumstellar disk (e.g. Quirrenbach et al. 1997). Be stars show no circumstellar dust (with only a few exceptions) and also lack molecular line emission and forbidden lines. These observations are consistent with the presence of high-density gas in a flattened disk.

In Fig. 1 we show three ISO-SWS spectra, of the LBV P Cygni, of the B[e] star HD 45677, and of the Be star $\gamma$ Cas, respectively (Lenorzer et al. 2002; see also Lamers et al. 1996a,b; Malfait et al. 1998; Hony et al. 2000 for detailed discussions of the ISO data). The spectra have been continuum subtracted and normalized to the line integrated flux of $\operatorname{Br} \alpha$ to facilitate comparision. The three spectra show a remarkable range in line flux behaviour. While the LBV shows very large line flux ratios between 


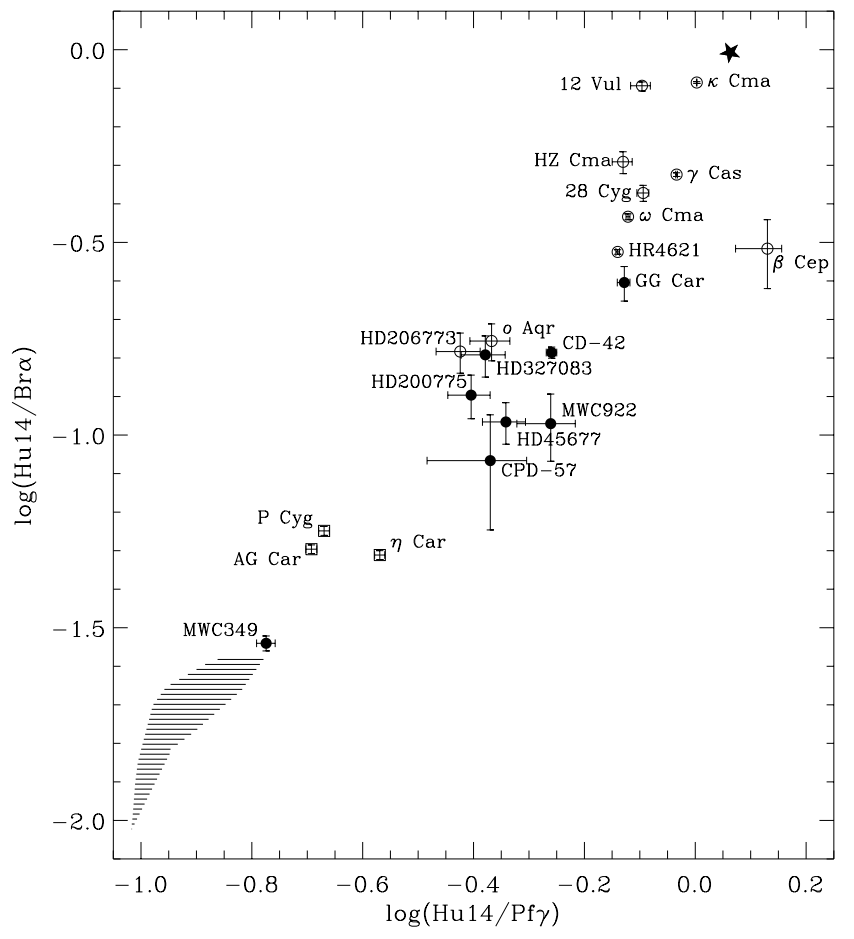

Fig. 2. $\mathrm{Hu}(14-6) / \mathrm{Br} \alpha$ versus $\mathrm{Hu}(14-6) / \mathrm{Pf} \gamma$ line ratio diagram for the hot stars observed with ISO (Lenorzer et al. 2002). The different classes of objects, LBVs (squares), B[e] stars (filled circles) and Be stars (open circles), are well separated. The thick asterisks indicates the position of optically thick black body emission; the striped region shows the range of ratios for Menzel case B recombination, including collisional de-excitation, for temperatures higher than $10^{4} \mathrm{~K}$.

lines, the $\mathrm{B}[\mathrm{e}]$ and Be stars do this to a much lesser extent. These observations suggest that if the line spectrum is mostly due to a stellar wind, the $\mathrm{H}$ I line fluxes roughly scale with the Einstein coefficients; this is much less the case in the $\mathrm{B}[\mathrm{e}]$ and Be star, where lines of very different intrinsic strength show similar line fluxes. This behaviour suggests that optical depths effects are important. The optical depth being dependent on the density squared, the line ratios are mainly probing the density distribution of the circumstellar material. Moreover, in the optically thick case the line ratios are probing the dependence in emitting surface with the wavelength which is also a function of the density stratification. In addition, non-LTE effects may play a modest role, as discussed by Hony et al. (2000).

It must therefore be possible to infer the density of the circumstellar material from the H I IR recombination lines without resolving them. In Sect. 3, we will present a simple diagram which quantifies this density probe.

\section{Line flux ratio diagnostic}

Figure 2 presents a diagram in which the line flux ratio of $\mathrm{Hu}(14-6) / \mathrm{Br} \alpha$ versus $\mathrm{Hu}(14-6) / \mathrm{Pf} \gamma$ is plotted for different types of emission line objects. The aim of this diagram is to provide a simple means to investigate the nature of circumstellar gas in highly obscured sources.
The diagram shows a clear trend in that both line flux ratios typically increase from $\mathrm{LBV}$ to $\mathrm{B}[\mathrm{e}]$ to Be stars. This trend can be understood in terms of the span in mass absorption coefficient between $\mathrm{Hu}(14-6), \operatorname{Pf} \gamma$ and $\operatorname{Br} \alpha$. In an optically thin medium one expects the plotted line flux ratios to follow Menzel Case B recombination theory. However, in an optically thick medium the plotted ratio becomes independent of mass absorption coefficient as the flux in any line is dominated by the size of the emitting surface. For optically thick lines the line flux ratio of two lines can be written as:

$\frac{I_{1}}{I_{2}}=\frac{B\left(\nu_{1}, T\right) S_{\mathrm{eff}, 1}}{B\left(\nu_{2}, T\right) S_{\mathrm{eff}, 2}}$

where $B(\nu, T)$ is the Planck function and $S_{\text {eff }}$ is the effective radiating surface in the line. If $S_{\text {eff }}$ is similar for both lines and the Rayleigh-Jeans approximation is applied, Eq. (1) reduces to: $I_{1} / I_{2}=\nu_{1}^{2} / \nu_{2}^{2}$.

This limit is indicated by a filled star in Fig. 2 and falls close to the locus of the Be stars. Note that we have assumed that the lines are in LTE and that the emitting medium is isothermal. Several Be stars deviate somewhat from the optically thick limit suggesting that there is a contribution from optically thin gas. This is to be expected since Be star disks can extend to $10-100 R_{*}$ and, depending on the density gradient in the disk, optically thin gas must contribute. Inclination may also play a role in that disks seen edge-on are expected to have a larger contribution from optically thin material than do disks seen face-on. Finally, it is known that disks of Be stars are of transient nature, their densities change with time, such that their position in the diagram is expected to vary more or less along the diagonal.

The locus of an optically thin isothermal gas emitting line radiation according to Menzel case B and for temperature greater than $10^{4} \mathrm{~K}$ is indicated by the dashed area in Fig. 2 (from Storey \& Hummer 1995). This region is close to that of the LBVs, suggesting that the bulk of the line emission from these stars is due to optically thin gas. This may be expected, since the winds are rapidly expanding and the density decreases as $r^{-2}$ or steeper (in the innermost regions). The high mass loss rate in a roughly spherical outflow causes a large emission measure and thus large line fluxes for the strongest lines, and a detectable signal even for the intrinsically much weaker lines.

The B[e] stars occupy a region in Fig. 2 which spans in between most of the Be and the LBV stars. If our interpretation of the line flux ratios in terms of densities is correct, this would imply that the $\mathrm{B}[\mathrm{e}]$ stars have contributions from both optically thick and thin regions to the line flux. This is qualitatively consistent with the notion that $\mathrm{B}[\mathrm{e}]$ stars have circumstellar disks (with high densities) but that the presence of forbidden line emission shows that an extended region of lower-density ionized gas must also be present. Perhaps the scale-height of the disks surrounding $\mathrm{B}[\mathrm{e}]$ stars is larger, or their disk radii are larger compared to the Be stars. 
It is interesting to discuss the location of some wellstudied, enigmatic objects in Fig. 2. The LBV $\eta$ Car is located near the Menzel case B limit, suggesting that the wind dominates the line emission from this star, and not the extended nebula. Detailed non-LTE model calculations indeed show that the UV to near-IR spectrum of $\eta$ Car can be fitted well using a very dense wind (Hillier et al. 2001). The peculiar star MWC349 is also located near the case B limit, again suggesting that the lines are formed in an optically thin medium. Polarimetry of MWC349 however shows that a dusty disk is present (Yudin 1996), but this disk is not contributing significantly to the emission from the ionized gas. This is consistent with the observed radio continuum spectrum of MWC349, which has the canonical $\nu^{0.6}$ slope expected for a spherically symmetric constant velocity wind (Rodriguez et al. 1986).

The example of MWC349 illustrates the care that needs to be taken when interpreting observations using our diagnostic diagram: stars close to the Menzel case B location may have a disk, however the $\mathrm{H}$ I lines are mainly not formed in the disk. The same care has to be taken when interpreting stars in the upper right corner of Fig. 2, where the line flux ratios are close to unity. For these, the conclusion must be that the lines are opaque and that the geometry of the main $\mathrm{HI}$ line emitting region is likely a disk. However, from the line flux ratio's alone one can not exclude geometries other than a disk, such as an optically thick (expanding) shell. Note that one may likely distinguish between these different geometries using kinematic information from resolved line profiles.

In first order, both $\operatorname{Pf} \gamma$ and $\operatorname{Br} \alpha$ probe the emission measure of the gas. This explains why the stars are moreor-less concentrated around the diagonal. So, in principle one can do the analysis using only one of the two line ratios. However, both lines have a specific advantage. The pro of $\mathrm{Br} \alpha$ is that it is the strongest line, while the advantage of $\mathrm{Pf} \gamma$ is that it suffers less from possible contamination by nebular emission. Plotting the line fluxes relative to $\mathrm{Hu}(14-6)$, the strongest Humphreys series line in the $L^{\prime}$-band, provides a better contrast between contributions from optically thin and thick material than does Br $\alpha$ relative to $\operatorname{Pf} \gamma$. We note that if reddening is significant it will affect $\mathrm{Hu}(14-6) / \mathrm{Pf} \gamma$ by shifting it to the right in the diagram by about $0.07 \times \log (\mathrm{Hu}(14-6) / \mathrm{Pf} \gamma)$ per magnitude of extinction. $\mathrm{Hu}(14-6) / \mathrm{Br} \alpha$ is not significantly affected as the wavelength separation between these two lines is very modest.

\section{Conclusion}

A considerable fraction of the massive stars of our galaxy is obscured by dust. Dust extinction is preventing us from using powerful criteria, developed at UV and optical wavelength ranges, to infer physical properties of hot stars. At wavelengths longwards of about 5 microns, emission from warm dust is typically dominating the spectra. It is therefore crucial to develop diagnostic tools in the near-infrared window in order to understand the nature and evolution of hot stars surrounded by dust, as it is the case, for instance, during the early stages of their lives in giant molecular clouds. The diagram presented in Fig. 2 provides such a tool. It gives a simple means to use line fluxes observed in the infrared $L^{\prime}$ window to constrain aspects of the density and spatial distribution of circumstellar gas around hot stars which may greatly help identifying the nature of such obscured sources.

Acknowledgements. We would like to thank S. Hony and C. Neiner for useful discussions, and B. Vandenbussche and P. Morris for invaluable help in obtaining and analysing the ISO observations. We acknowledge support from an NWO "Pionier" grant. This work was partly supported by NWO Spinoza grant 08-0 to E. P. J. van den Heuvel.

\section{References}

Bjorkman, K. S., Miroshnichenko, A. S., Bjorkman, J. E., et al. 1998, ApJ, 509, 904

Castor, J. I., \& Lamers, H. J. G. L. M. 1979, ApJS, 39, 481

Chesneau, O., Roche, M., Boccaletti, A., et al. 2000, A\&AS, 144,523

Dachs, J., Hanuschik, R., \& Kaiser, D. 1986, A\&A, 159, 276

van Genderen, A. M. 2001, A\&A, 366, 508

de Graauw, T., Haser, L. N., Beintema, D. A., et al. 1996, A\&A, 315, L49

Hillier, D. J., Davidson, K., Ishibashi, K., \& Gull, T. 2001, ApJ, 553, 837

Hony, S., Waters, L. B. F. M., Zaal, P. A., et al. 2000, A\&A, 355,187

Humphreys, R. M., \& Davidson, K. 1994, PASP, 106, 1025

Kessler, M. F., Steinz, J. A., Anderegg, M. E., et al. 1996, A\&A, 315, L27

Lamers, H. J. G. L. M, Morris, P. W., Voors, R. H. M., et al. 1996a, A\&A, 315, 225

Lamers, H. J. G. L. M, Najarro, F., Kudritzki, R. P., et al. 1996b, A\&A, 315, 229

Lamers, H. J. G. L. M., Zickgraf, F.-J., de Winter, D., Houziaux, L., \& Zorec, J. 1998, A\&A, 340, 117

Lenorzer, A., Vandenbussche, B., Morris, P., et al. 2002, A\&A, 384,473

Limber, D. N., \& Marlborough, J. M. 1968, ApJ, 152, 181

Malfait, K., Waelkens, C., Waters, L. B. F. M., et al. 1998, A\&A, 332, 25

McGregor, P. J., Hyland, A. R., \& McGinn, M. T. 1989, A\&A, 223, 237

Quirrenbach, A., Bjorkman, K. S., Bjorkman, J. E., et al. 1997, ApJ, 479, 477

Rodriguez, L. F., Canto, J., Escalante, V., \& Moran, J. M. 1986, in NASA, Ames Research Center Summer School on Interstellar Processes, 17

Skinner, C. J., Exter, K. M., Barlow, M. J., Davis, R. J., \& Bode, M. F. 1997, MNRAS, 288, L7

Storey, P. J., \& Hummer, D. G. 1995, MNRAS, 272, 41

Struve, O. 1933, ApJ, 77, 66

Swings, J. P. 1974, A\&A, 34, 333

Vakili, F., Mourard, D., Bonneau, D., Morand, F., \& Stee, P. 1997, A\&A, 323, 183

Yudin, R. V. 1996, A\&A, 312, 234 